\title{
Sequential delivery of therapeutic agents using a rationally designed disulfide-linked glycolipid-like nanocarrier
}

\author{
Yingwen $\mathrm{Hu}^{1,2}$, Na Liu ${ }^{1}$, Bolin Cheng ${ }^{1}$, Yanan Tan ${ }^{1}$, Lijuan Wen ${ }^{1}$, Hong Yuan ${ }^{1}$, \\ Fuqiang $\mathrm{Hu}^{1}$ \\ ${ }^{1}$ Institute of Pharmaceutics, College of Pharmaceutical Sciences, Zhejiang University, Hangzhou 310058, People's Republic \\ of China \\ ${ }^{2}$ Department of Chemistry, Purdue University, West Lafayette, IN 47907, United States of America \\ Correspondence to: Fuqiang Hu, email: hufq@zju.edu.cn \\ Keywords: co-delivery, sequential, combined therapy, redox-responsive, drug resistance \\ Received: January 20, $2016 \quad$ Accepted: October 13, $2016 \quad$ Published: November 04, 2016
}

\section{ABSTRACT}

\begin{abstract}
Usage of combination therapies to deliver multiple therapeutics to increase treatment efficacy has shown promising results in the clinic. In an effort to maximize the synergistic effect of co-delivery of a drug and siRNA, we have developed a timedependent sequential drug delivery system (DDS) based on a disulfide-linked chitosanbased nanocarrier (CS-sS-SA) for the co-delivery of paclitaxel (PTX) and Bcl-2 specific siRNA (siBcl-2). This CS-ss-SA nanocarrier is able to transport both drug and siRNA by entrapment of PTX and adsorption of siRNA on the shell by electrostatic attraction. We show that this nanocarrier transports siRNA into tumor cells via its glycolipidlike spatial structure and releases a hydrophobic model drug, Nile Red 8-11 h later. Next, when siRNA and the hydrophobic drug PTX were co-delivered to tumor cells, a synergistic effect was observed in both cell cycle arrest and cell viability. Ultimately, the co-delivery of PTX and siBcl-2 by CS-ss-SA may prove to be more efficacious and may even help overcome drug resistance.
\end{abstract}

\section{INTRODUCTION}

In recent years, advances in nanotechnology and biotechnology have opened up unprecedented opportunities for controlled drug delivery and novel codelivery strategies $[1,2]$. With a rational design, a drug combination with different therapeutic agents in the same delivery system can allow significant benefits in cancer therapy, including reduced therapeutic doses, side effects, development of drug-resistance and, in the long run, cost [3-6]. In particular, through gene silencing, a window can open where the tumor cells are transiently sensitized to a cytotoxin. Therefore, sequential delivery of siRNA and a conventional cytotoxin in a single nanocarrier offers considerable potential for improving therapeutic outcomes [7-10].

However, it should be noted that the chemotherapeutics and RNAi combination therapy still faces significant challenges to fulfill potential clinical applications. First, the optimized timing/sequence of release of the cytotoxic and siRNA agents from the system, which is a key factor to achieve the best synergistic outcome is not well understood. The ideal codelivery carrier with an optimized drug releasing sequence should first release the siRNA to achieve knock-down of the target gene, leading to a transient window of increased cytotoxin sensitivity. The ideal co-delivery carrier would only then release the cytotoxin, for maximum efficacy [11, 12].

According to these principles, we proposed a disulfide-linked glycolipid-like nanocarrier (chitosanSS-stearic amine, CS-ss-SA) as the co-delivery system. Chitosan, with a positive zeta potential has been demonstrated to compact oligonucleotides readily on its shell [13]. With the particular spatial structure of the glycolipid-like nanocarrier, hydrophobic drugs can be easily encapsulated in their inner cores, which makes it possible to deliver both nucleotide and small molecule drugs in one regimen $[14,15]$. With the selective redox responsive disulfide linker, the shell will be detached upon internalization, releasing the compacted siRNA. Subsequently, the cytotoxin will gradually release from 
the core into the cytoplasm, exerting its cytotoxic effects. Therefore, this nanocarrier would allow for the sequential delivery of therapeutic agents.

In this manuscript, we report the development of a glycolipid-like nanocarrier based on chitosan (CS-SS-SA) for the co-delivery of a Bcl-2 specific siRNA ( $\mathrm{siBcl}-2$ ) and the hydrophobic antitumor drug paclitaxel (PTX). We studied the cellular pathway of the co-delivery system, including internalization, endo-lysosome escape and sequential drug release against the MCF-7 breast cancer cell line. Then we investigated cell cycle arrest and loss of cell viability induced by the co-delivery of PTX and siBcl-2 to normal and multi-drug resistant MCF-7 cell lines. We demonstrate time dependent sequential drug delivery achieves synergetic effects and potentially can be used to treat drug resistant cell lines (Scheme 1).

\section{RESULTS AND DISCUSSION}

\section{Preparation and characterization of co-delivery core/shell complexes}

The nano-carrier CS-ss-SA was prepared using a previously reported procedure [16]. SA was crosslinked with chitosan by a two-step amide coupling to produce CS-ss-SA. The amino substitution ratio (SD\%) was determined as $9.57 \%$ (molar ratio), which provided the molecular weight of the final polymer as approximately $18.9 \mathrm{KDa}$.

To prepare the disulfide-linked glycolipid-like co-delivery complexes, first, paclitaxel was loaded into Chitosan-SS-steraic acid (CS-ss-SA) with approximately $80 \%$ loading efficiency. Next, siRNA was electrostatically bound to the positive amine groups on the chitosan shell of the drug-loaded nanoparticles. The hydrodynamic size of the co-delivery core/shell nanoparticles was measured to be $160.0 \pm 37.0 \mathrm{~nm}$ by DLS (Figure 1a) and the size measured by TEM was approximately 140 $\mathrm{nm}$ (Figure 1b), which showed increased particle size than only drug-loaded nanoparticles (Figure S1). The zeta potential of the co-delivery complexes was $15.6 \pm$ $2.8 \mathrm{mV}$ (Figure 1c), which was lower than that of only drug-loaded nanoparticles $(33.9 \pm 1.3 \mathrm{mV})$ because of the incorporation of negatively charged siRNA on the shell. The binding ability of the complexes was assessed using a gel retardation assay (Figure $1 \mathrm{~d}$ ). When the N/P ratio of complexes reached 75 , the migration of siRNA was completely retarded, suggesting that stable complexes were formed when the N/P ratio was at or above 75. Since siRNA is readily enzymatically degraded by RNases, a critical advantage of nanocarrier-mediated delivery is the ability to prevent premature destruction of siRNA [14]. In order to assess the capacity of CS-ss-SA to protect siRNA from degradation by RNases, CS-ss-SA/siRNA complexes

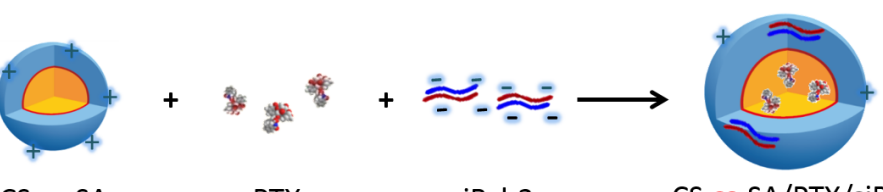

$\begin{array}{llll}\text { CS-ss-SA } & \text { PTX } & \text { SiBcl-2 } & \text { CS-ss-SA/PTX/siBcl-2 }\end{array}$ complex

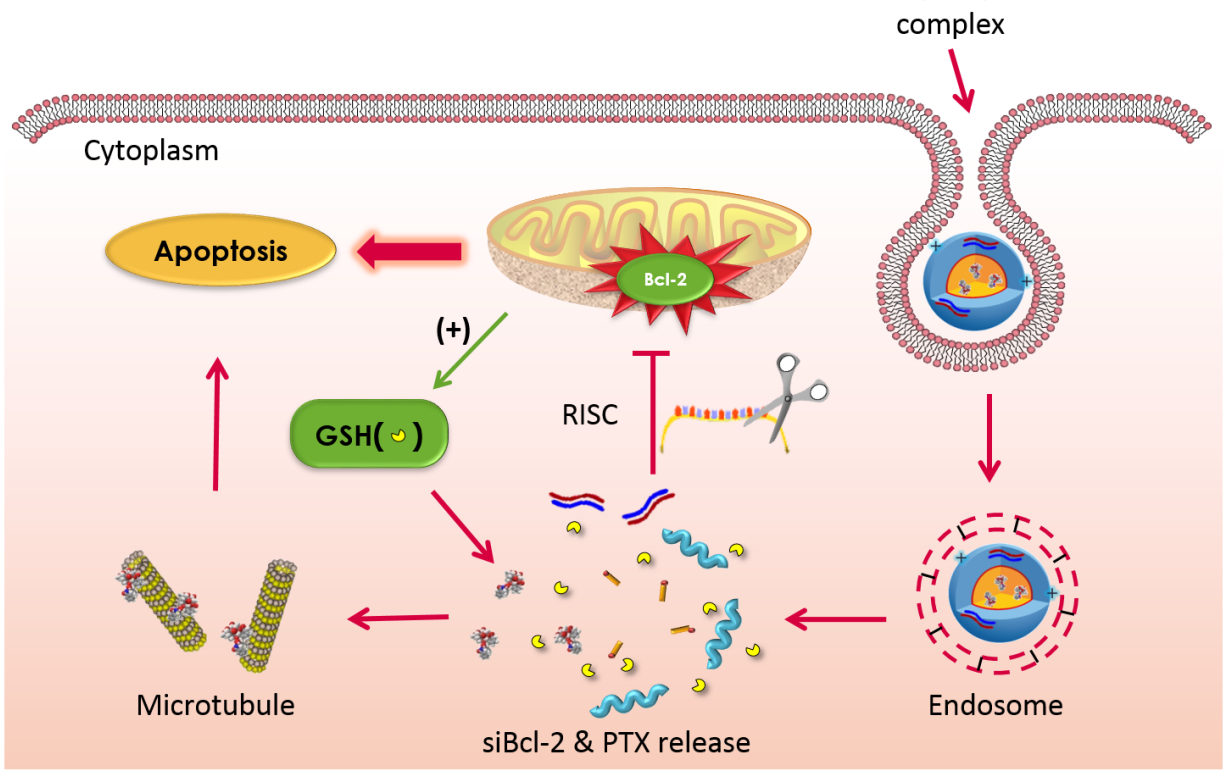

Scheme 1: The schematic diagram of the co-delivery system. Schematic structure of siRNA targeted to Bcl-2 (siBcl-2) and paclitaxel (PTX) loaded CS-ss-SA complexes, which was fast uptake by tumor cells, responded to the endogenous high GSH in tumor cells and sequentially released the siRNA and PTX to perform maximized cytotoxicity. 
were prepared with N/P ratios $=75$ and incubated with a solution of RNase A. Degradation of siRNA was visualized via gel electrophoresis and the results are presented in Figure S2. Free siRNA was completely digested with no full-length band observed, while clear migration of the full-length siRNA band was observed even after RNase treatment. These results indicate that CS-Ss-SA is able to effectively protect siRNA from RNase degradation.

\section{In vitro siRNA release}

In order to release the siRNA from the chitosan shell, the disulfide bonds must be reduced to free thiols, which disrupt the overall structure of the DDS. In order to tune the sensitivity of the disulfide reduction such that the siRNA is not prematurely released before the DDS reaches its target, the structural characteristics of the DDS were modified. In previous studies, we have prepared CS-ss-SA with a range of graft ratios [16]. We found that the introduction of additional disulfide linkers decreased the sensitivity to cleavage in higher reducing environments. Using this rational design strategy, CS-ssSA could degrade correspondingly to different levels of reducing environment, release the payloads, and be used for triggered-release in different tumor types. In this study, we used the optimal ratio of disulfide linkers to construct the CS-SsSA ${ }_{20 \%}$ which should only release their siRNA cargo in highly reducing environments. To determine the rate of release, fluorescently labeled siRNA was adsorbed onto CS-ss-SA ${ }_{20 \%}$ nanoparticles. These nanoparticles

$\mathbf{a}$
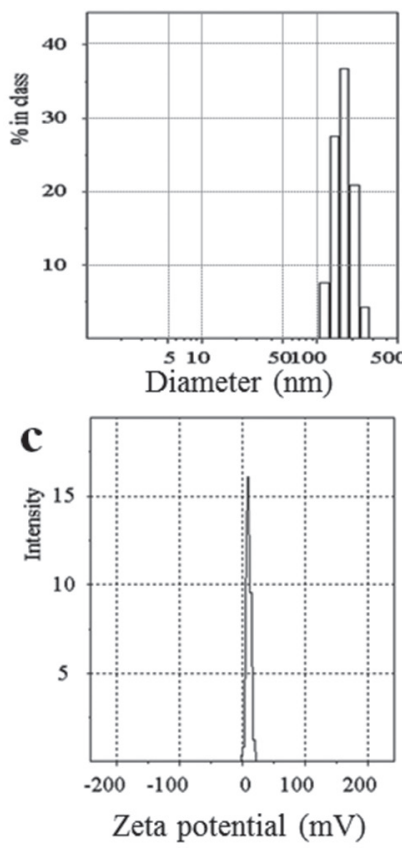

were incubated in non-reducing and reducing $(10 \mathrm{mM}$ GSH) environments. As shown in Figure S3, fluorescence intensity (I) and FAM-siRNA concentrations (C) showed a linear regression: $\mathrm{I}=37.08 \times \mathrm{C}-0.62, \mathrm{r}^{2}=0.9995$, which confirmed to be $0.165-13.2 \mu \mathrm{g} / \mathrm{mL}$. The disassociation of the siRNA from the CS-ss-SA/FAM-siRNA complex was fairly rapid with a cumulative release of $78.2 \%$ in $8 \mathrm{~h}$, when in the presence of $10 \mathrm{mM}$ GSH. While in the GSHfree release medium, siRNA release rate was relatively slow in which the cumulative release was less than $50 \%$ at $12 \mathrm{~h}$. The difference in release rates is most likely due to the weakening of the electrostatic interactions between chitosan and siRNA upon reduction of the nanoparticle chemical backbone.

\section{Cellular uptake and endo-lysosome escape}

To track complexes after cellular uptake and to evaluate their endo-lysosome escape capacity, the intracellular distribution of CS-SS-SA/siRNA complexes in MCF-7 cells was investigated by confocal laser scanning microscopy (CLSM) (Figure 2). Co-localization of the fluorescent FAM-siRNA (green) with Lysotrackerstained lysosomes (blue) produced cyan fluorescence in the merged images. At 1h, large numbers of cyan pixels were observed in treated cells with $82.4 \%$ of siRNA colocalized with lysosomes. This result indicates the CSss-SA/siRNA complexes were trapped in the lysosomes. After $4 \mathrm{~h}$, fewer cyan pixels were observed. After $12 \mathrm{~h}$, only $8.03 \%$ of siRNA was co-localized with lysosomes,

b

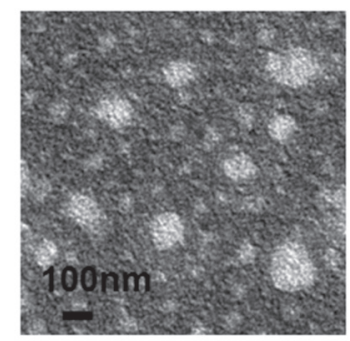

d Free $\frac{\text { N/P ratio }}{\text { siRNA }} \underset{510255075100}{1025 \quad}$

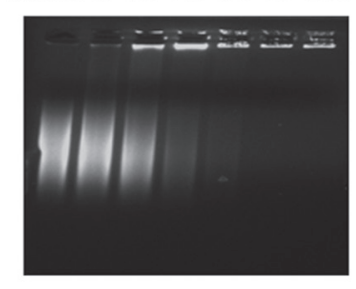

Figure 1: Characteristics of CS-ss-SA/PTX/siRNA complexes: a. size distribution. b. TEM observation, c. zeta potential, d. gel retardation analyses of complexes with different $\mathrm{N} / \mathrm{P}$ ratios. 
suggesting that most of the complexes have escaped. These observations may be due to the ionization state of chitosan at tumor acidic endo-lysosome environment ( $\mathrm{pH} 4.5-6.4)$, which is near its $\mathrm{pKa}$ (5-7 for chitosan and independent on the degree of ionization), which may increase endosomal $\mathrm{pH}$ leading to endosomal release [13, 21].

\section{Intracellular triggered siRNA release}

To further characterize the intracellular siRNA release, a FRET assay and molecular beacons (MB) were applied. Real-time monitoring of redox-responsive complexes in the presence of high/low levels of GSH in MCF-7 cells was enabled.

In this study, non-coding siRNA labeled with FAM (FAM-siNC, $\mathrm{E}_{\mathrm{x}}=487 \mathrm{~nm}$ ) and the cationic vector CS-ssSA labelled with RITC (RITC-CS-ss-SA, E $=546 \mathrm{~nm}$ ) were used as the FRET donor and receptor, respectively. These two dyes were chosen because of their superior photostability, large spectrum overlaps and high FRET efficiency.

Buthionine sulfoximine (BSO) was added to lower the intracellular GSH concentration according to literature and untreated MCF-7 cells were used as a control [22]. RITC-CS-ss-SA/FAM-siRNA complexes were incubated with BSO pre-treated or untreated cells. FRET eff $_{\text {( }}(\%)$ between the donor and acceptor as a function of time is shown in Figure 3. When the receptor (RITC) was bleached in the region, fluorescence intensity of the donor (FAM, green) rapidly increased, indicating significant FRET between the two fluorophores (Figure 3a). As seen in Figure $3 \mathrm{~b}$, after $1 \mathrm{~h}$ of incubation, the different GSH concentrations made no significant difference in the FRET $_{\text {eff }}$ of the MCF-7 cells. In $2 \mathrm{~h}$, the FRET $_{\text {eff }}$ of untreated MCF-7 cells decreased significantly $(p=0.032)$. After 12 $\mathrm{h}$ of incubation, the FRET $_{\text {eff }}$ of untreated MCF-7 cells decreased even further to below $30 \%$, indicating siRNA release from the complexes during the $12 \mathrm{~h}$. Whereas the $\mathrm{FRET}_{\text {eff }}$ of BSO pre-treated cells maintained a high percentage $(\sim 50 \%)$, which illustrated that the release of siRNA from the complexes was significantly slower with a lower GSH concentration in the BSO pre-treated cells.

Molecular beacon (MB) is a dual-labeled hairpin oligonucleotide probe comprising a fluorophore and a quencher at opposite ends. With the stem-loop structure, the fluorescence of the fluorophore remains quenched in the absence of a complementary target. When the

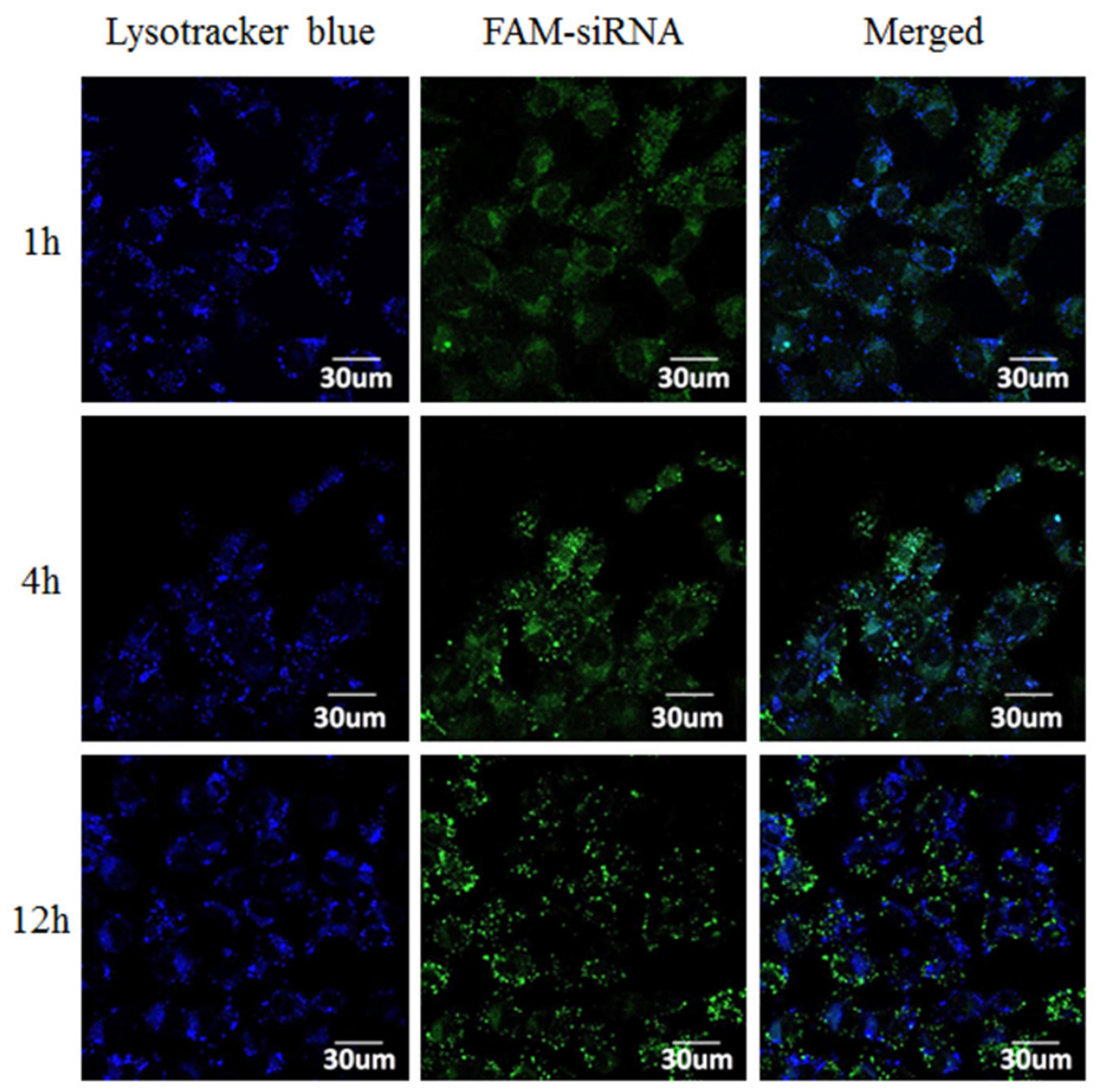

Figure 2: Confocal images of intracellular trafficking of the complexes and lysosome on MCF-7 cells. Lysotracker-labelled lysosomes (blue), FAM-labelled siRNA (green), and the merged images. 
molecular beacon hybridizes with its target, the hairpin structure will open, allowing the fluorescence signal to be emitted [23, 24]. Based on these principles, a Cy5labeled molecular beacon targeting the human GAPDH (glyceraldehyde 3-phosphate dehydrogenase) GAPDH$\mathrm{MB}$ (Cy5-MB) was utilized in this study to transduce the oligonucleotide release from the CS-Ss-SA/Cy5-MB complexes directly into a fluorescence signal by flow cytometry.

Fluorescence intensity of untreated and BSOpretreated MCF-7 cells as a function of time was shown in Figure S4. The fluorescence intensity of untreated group increased gradually over time, which indicated that with the high GSH concentration in the tumor cells, Cy5-MB fast released from the CS-ss-SA/Cy5-MB complex, bind to the target GAPDH sequence, and emitted fluorescence. Whereas in the BSO-pretreated group, intracellular fluorescence intensity showed no significant change over time. The fluorescence intensity of the untreated group was 39 times higher than that of the BSO-pretreated group, indicating that the amount of free MB in the BSOpretreated cells dissociated from the complex was only $2.6 \%$ of the untreated group.

These results demonstrated that in response to the high concentration of reducing agents, CS-ss-SA complexes can readily release the siRNA/oligonucleotides into tumor cells. When the GSH concentration was decreased by BSO in the tumor cells, the release rate significantly decelerates.

\section{Intracellular sequential drug delivery}

The biggest challenge in co-delivery is to find applicable carriers to optimize the timing/location of release of chemotherapy and siRNA agents in tumor cells. The incorporation of additional drug payloads affects the pharmacokinetics of the nanocarrier and requires significant modifications of the carrier design. We further studied the intracellular sequential drug delivery of complexes in MCF-7 cells. Nile red (NR) and FAMlabeled non-coding siRNA ( siNC) were used as the model drugs and fluorescent probes.

NR was chosen for its unique spectral signature, where the emitted signal is dependent upon the hydrophobicity of its environment. When NR became trapped inside the nanoparticle, its fluorescence intensity at this wavelength essentially disappeared (Figure S5).

The cells underwent incubation with CS-ss-SA/ NR/FAM-siNC complexes for periods of 1, 4, 8, and 12 $\mathrm{h}$ respectively and intracellular fluorescence images were observed to monitor the drug delivery behavior. As shown in Figure 4, large numbers of green fluorescent bodies (FAM-siNC) were observed in the first hour, indicating the complexes first released the siRNA cargo nearly immediately after cellular internalization. The majority of visible red fluorescent bodies (NR) started to appear after the $8 \mathrm{~h}$ timepoint, which shows the distinctly different release kinetics of the two cargoes with the NR clearly being released 7 to 11 hours later than the siRNA.

\section{In vitro siRNA transfection}

MCF-7 cells were incubated with CS-ss-SA/siBcl-2 complexes for $24 \mathrm{~h}$ and then intracellular Bcl-2 mRNA levels were determined by RT-PCR. After sequencespecific Bcl-2 silencing by CS-ss-SA/siBcl-2 complexes, reduced Bcl-2 mRNA levels were observed (Figure 5a). Elevated siBcl-2 concentrations produced increased gene knockdown, with 50, 100 and $200 \mathrm{nM}$ of siBcl-2 by CSss-SA/siBcl-2 complexes inducing 79.4\%, 85.8\% and 93.1\% knockdown of Bcl-2 mRNA. In comparison, the negative control showed neglible knockdown efficiency. Transfection using the CS-Ss-SA/siBcl-2 complexes is similar to that of the Lipofectamine ${ }^{2000}$ transfection reagent
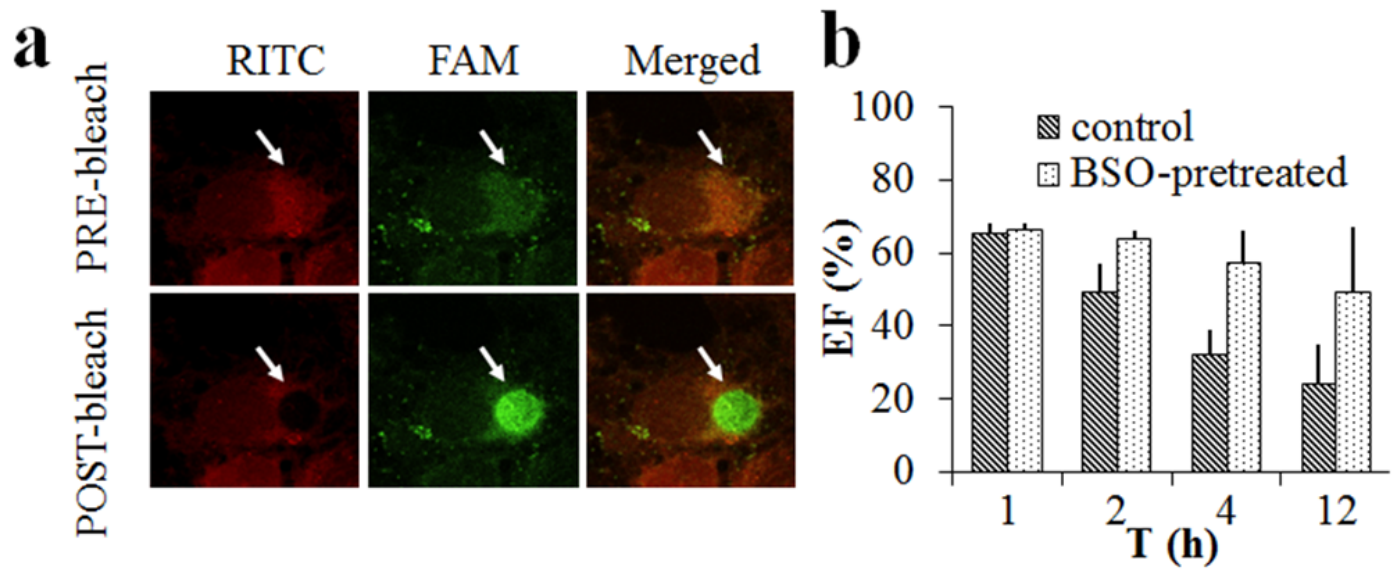

Figure 3: FRET images on the MCF-7 cells incubated with RITC-labelled CS-ss-SA/FAM-siRNA complexes. a. FRET images before and after acceptor bleaching in fixed cells, from left to right: RITC-labeled CS-ss-SA, FAM-labeled siRNA, and merged channel. b. Quantitative determination of FRET $_{\text {eff }}$ on the BSO-pretreated or untreated MCF-7 cells in $12 \mathrm{~h}$. 
carrying the same dose, indicating that the gene vector is highly effective.

\section{Cell cycle analysis}

Overexpression of $\mathrm{Bcl}-2$ protein is related to drug resistance and poor prognoses in cancer patients [25].
Simply knocking down Bcl-2 has not been found to effectively suppress cancer activity, most likely due to the complexity of the apoptotic signaling pathway [26-29]. According to the mechanism of drug action, the silencing of the Bcl-2 gene will open a window of time in which the cell population's cell cycle is transiently synchronized, becoming more sensitive to chemotherapy [11, 30]. As

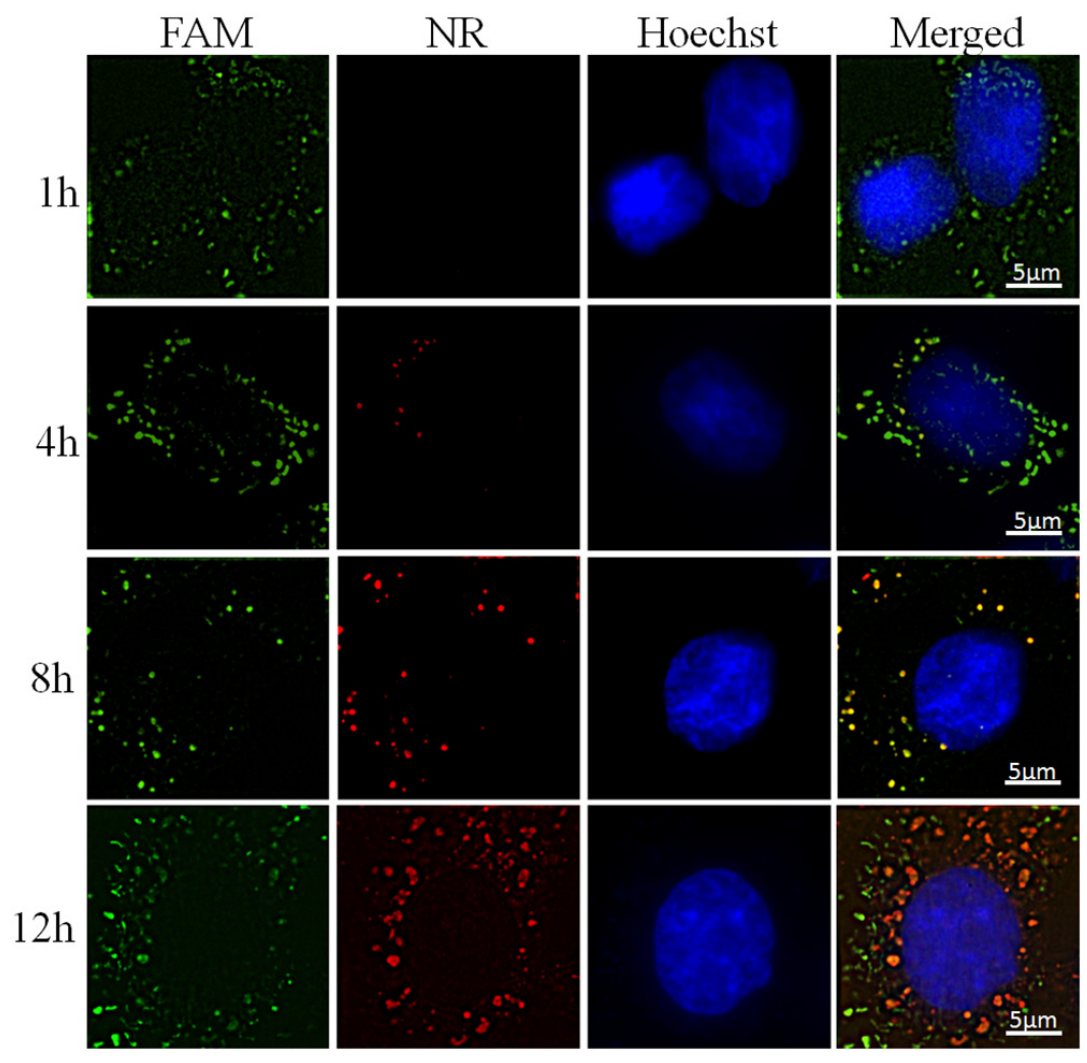

Figure 4: Sequential delivery FAM-siNC and NR in MCF-7 cells after incubated with CS-ss-SA/NR/FAM-siNC complexes for $\mathbf{1 ,}, \mathbf{4}, \mathbf{8}$, and $12 \mathrm{~h}$. From left to right, FAM-labeled non-coding siRNA, Nile Red, Hoechst labeled nuclei and merged channel.

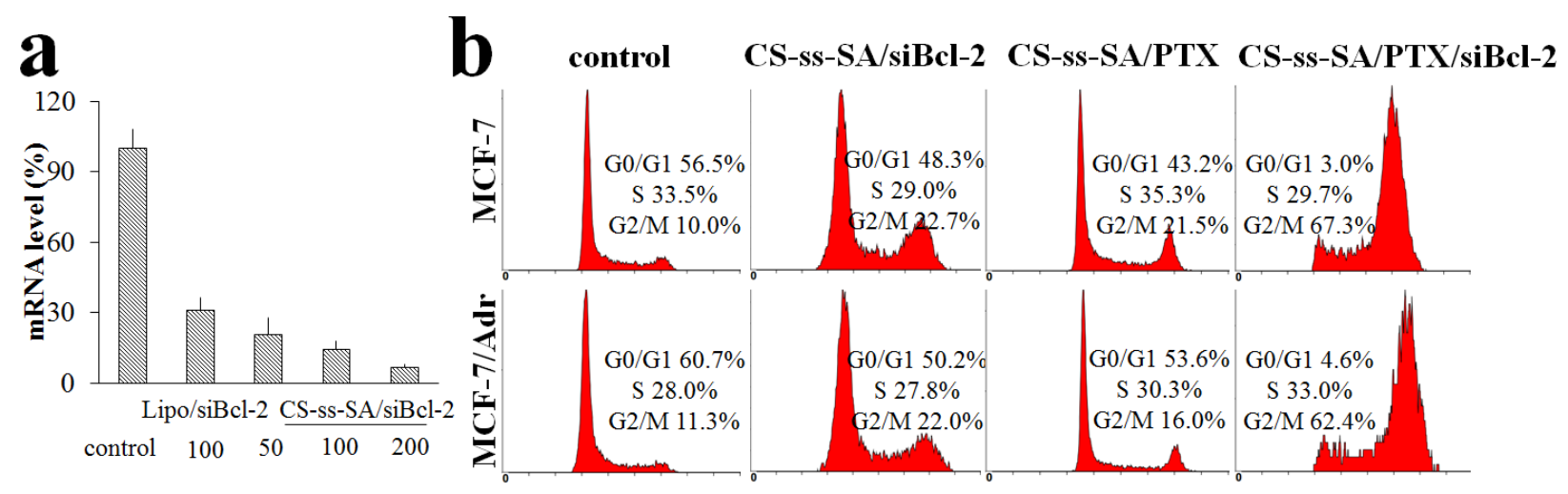

Figure 5: a. Bcl-2 mRNA levels regulation in MCF-7 cells by the transfection of CS-ss-SA/siBcl-2. b. Cell cycle distributions of MCF-7 and MCF-7/Adr cells incubated with CS-ss-SA/siBcl-2, CS-ss-SA/PTX and CS-ss-SA/PTX/siBcl-2 complexes (siBcl-2 100 nM, PTX 0.1 $\mu \mathrm{g} / \mathrm{mL}$ ) for $48 \mathrm{~h}$. 
one of the most frequently used drugs in the treatment of breast cancer, dose-dependent side effect of PTX greatly limits its clinical application [31]. Thus, reducing the PTX dose or increasing the sensitivity of tumor cells is of particular importance. PTX interferes with mitotic spindle function through tubulin polymerization and arrests the $\mathrm{G} 2 / \mathrm{M}$ phase of cell cycle.

In this study, CS-ss-SA/siBcl-2/PTX complexes were incubated with MCF-7 cells and multi-drug resistant MCF-7/Adr cells, which introduced G2/M cell-cycle arrest after $48 \mathrm{~h}$ (Figure 5b). In MCF-7 cells, G2/M population gradually increased from $10.0 \%$ to $21.5 \%$, indicating that PTX-loaded CS-ss-SA nanoparticles release PTX. Whereas after incubation with CS-ss-SA/siBcl-2/PTX complexes, the G2/M population increased significantly to $67.3 \%$, indicating that co-delivery of siBcl-2 and PTX was more efficacious. A similar tendency was also seen in MCF-7/Adr cells, indicating the possibility of the synergetic effect induced by the co-delivery system against both drug-sensitive and resistant cells.

\section{Synergy quantification of the co-delivery complexes}

Cytotoxicitystudies were performed to probe the potential synergistic effect of the co-delivery system to downregulate the apoptosis threshold and overcome the drug resistance of MCF-7 and MCF-7/Adr cells. The experiments were designed to gain insight into the quantitative measurement of Bcl-2 silencing and PTX to exert their maximum effect. As shown in the Figure 6a-6c, CS-ss-SA micelles exhibited negligible toxicity in MCF7 cells, while CS-ss-SA/siBcl-2/PTX complexes showed a high cytotoxicity (74.9\%). The co-delivery CS-ss-SA/ siBcl-2/PTX complexes presented significantly higher cytotoxicity than that of CS-ss-SA/PTX nanoparticle $(39.1 \%, \mathrm{p}=0.0023)$ or $\mathrm{CS}-\mathrm{ss}-\mathrm{SA} / \mathrm{siBcl}-2$ complexes $(28.6 \%, p=0.0082)$ alone. Since the concentration of PTX in the experiment was $0.1 \mu \mathrm{g} / \mathrm{mL}$, the greatly increased cytotoxicity was possibly attributed to the synergistic effect of PTX and silencing of Bcl-2 gene.

To further confirm the synergistic assumption of the co-delivery system, we used the the ChouTalalay combination index (CI) method to quantitate the synergistic effect [32]. A free computer software "Calcusyn" provided by Prof. Chou was used for data analysis. The software generated the simulation and provided PD parameters (Dm and $\mathrm{m}$ ), curves for doseeffect, the Fa-CI and median effect plot, and the Summary Table. The CI values of the co-delivery system as a function of the cell viability was simulated (Figure 6). In Figure $6 \mathrm{~b}$ and $6 \mathrm{e}, 6 \mathrm{D}$ represents the drug dose, fa is affected fraction, while fu is the unaffected fraction. Dm represents the half maximal inhibitory concentration
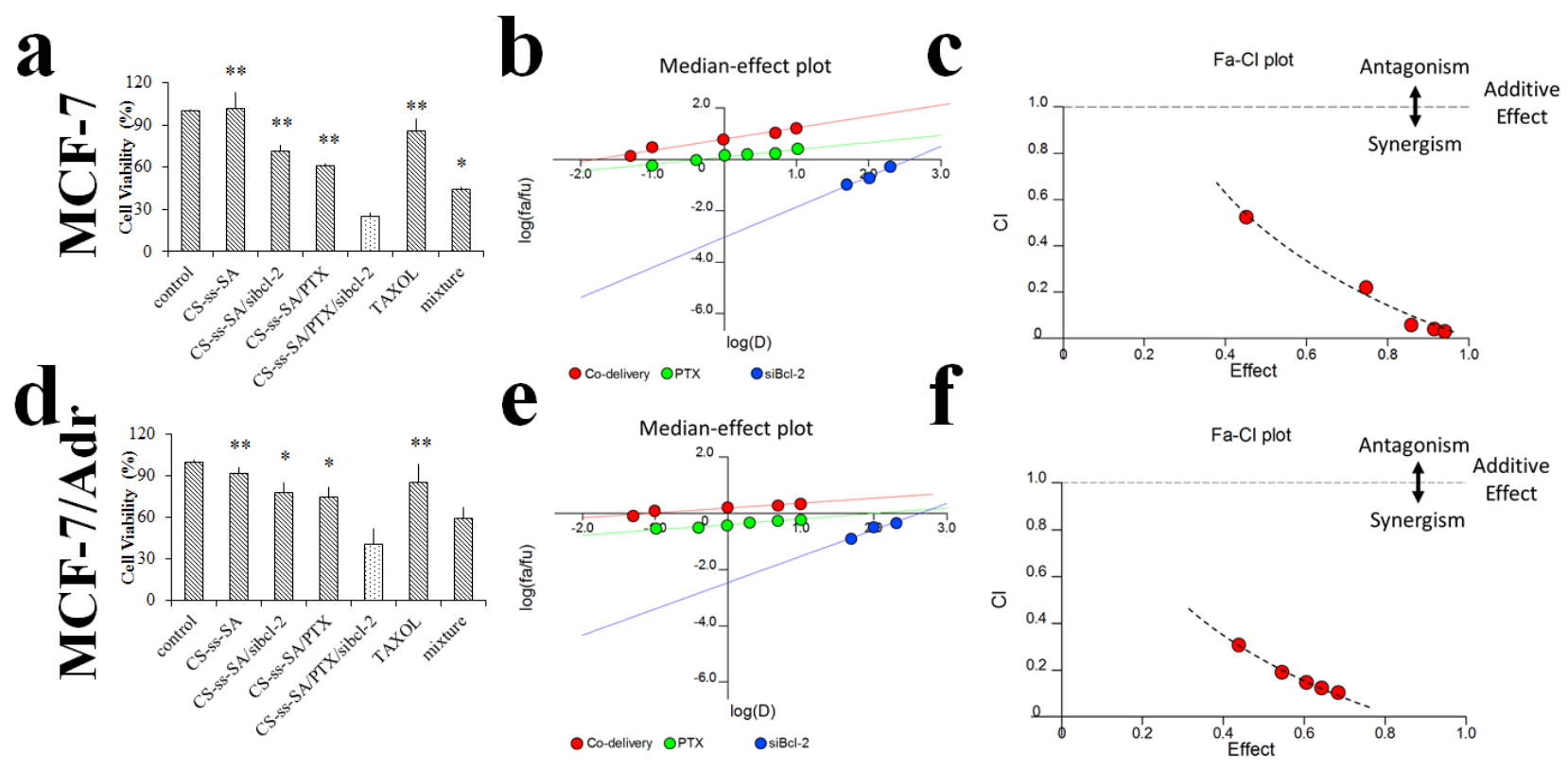

Figure 6: Cytotoxicity of the co-delivery complexes. a, d. Cell viability of the co-delivery of siBcl-2 and PTX by CS-ss-SA against MCF-7 and MCF-7/Adr cells. The concentration of PTX was $0.1 \mu \mathrm{g} / \mathrm{mL}$, while the concentration of siBcl-2 was $100 \mathrm{nM}$. b, e. Medianeffect plot. " $\mathrm{D}$ " represents the dose (or concentration) of a drug, fa is the fraction affected by $\mathrm{D}$, and fu is the fraction unaffected (i.e., fu $=1-\mathrm{fa}$ ). Dm is the median-effect dose ( $\mathrm{IC}_{50}$ in this study) that inhibits the system under study by $50 \%$, and $\mathrm{m}$ is the coefficient signifying the shape of the dose-effect relationship, where $\mathrm{m}=1,>1$, and $<1$ indicate hyperbolic, sigmoidal, and flat sigmoidal dose-effect curves, respectively. c, f. Plot of the combination index (CI) as the function of cell viability. Untreated cells were used as control. The y-axis (CI) was a function of effect levels (fa) on the x-axis. CI was introduced for quantification of synergism or antagonism for two drugs, where CI $<1,=1$, and $>1$ indicate synergism, additive effect, and antagonism, respectively. 
$\left(\mathrm{IC}_{50}\right)$, and $\mathrm{m}$ is the coefficient. CI was introduced for quantification of synergistic or antagonistic effects for the two drugs:

$$
\begin{aligned}
C I= & \frac{(D)_{1}}{\left(D_{x}\right)_{1}}+\frac{(D)_{2}}{\left(D_{x}\right)_{2}}=\frac{(D)_{1}}{\left(D_{m}\right)_{1} f_{a} /\left(1-f_{a}\right)^{1 / m_{1}}}+ \\
& \frac{(D)_{2}}{\left(D_{m}\right)_{2} f_{a} /\left(1-f_{a}\right)^{1 / m_{2}}}
\end{aligned}
$$

where $\mathrm{CI}<1$ or $>1$ indicate a synergistic or antagonistic effect, respectively.

In this study, calculated CI values were established for doses providing a 50,75, and $90 \%$ cancer cell-killing effect $\left(\mathrm{ED}_{50}, \mathrm{ED}_{75}\right.$ and $\left.\mathrm{ED}_{90}\right)$. The results for $\mathrm{ED}_{90}$ revealed that co-delivery of PTX and siBcl-2 with all doses had CI values between 0.29 to 0.03 , indicating a strong synergistic effect (Figure 6c). For the drug resistant MCF$7 /$ Adr cells, the CI values ranged from 0.31 to 0.10 , also indicating a significant synergism (Figure 6f). Based on these results, we conclude that the co-delivery complexes had synergistic effects, and showed the capacity of overcoming multi-drug resistance.

Interestingly, the co-delivery system showed higher cell growth inhibition than that of the mixture of PTX-loaded nanoparticles and siRNA-bound complexes administered at the same dose. Reports have shown that siRNA and chemotherapeutic agents co-delivered in the same carrier system had a greater inhibitory effect on cell proliferation than delivered separately in different carriers [33-35]. This is mainly because the same drug delivery system with chemotherapeutics and siRNA guaranteed similar cell internalization and intracellular sequential drug release for both treatments in the tumor cells. For long-term consideration, a co-delivery system with the same carrier could guarantee similar pharmacokinetics and concomitant passive accumulation of both treatments in the same tissue and cells $[1,36]$.

\section{CONCLUSION}

In conclusion, we have designed and applied an effective co-delivery nanocarrier for an optimized synergetic effect. Significantly, the release of siRNA was correlated positively with the intracellular GSH level. SiRNA and a hydrophobic drug were sequentially delivered into cells by a nanocarrier and generated cell cycle arrest and cytotoxicity in breast cancer cells and drug-resistant cells. This co-delivery system will facilitate the rational design of a sequential combined therapy.

\section{MATERIALS AND METHODS}

\section{Materials}

Chitosan with an approximate $15.0 \mathrm{kDa}$ average molecular weight was obtained by enzymatic degradation of $95 \%$ deacetylate chitosan $(\mathrm{Mw}=450 \mathrm{kDa})$ and was supplied by Yuhuan Marine (Yuhuan, China). Stearic acid (SA) was purchased from Fluka (Milwaukee, WI, USA). 3, 3'-dithiodipropionic acid was purchased from Tokyo Chemical Industry (Tokyo, Japan). FAM-siRNA (FAM5'-UUCUCCGAACGUGUCACGUTT-3), Non-coding siRNA (siNC, 5'-UUCUUCGAACGUGUCACGUTT-3), and Bcl-2 specific siRNA (siBcl-2, 5'-CCCUGUGGAUGACUGAGUATT-3) was purchased from Shanghai GenePharma (Shanghai, China). Paclitaxel (PTX) was purchased from Shanghai Zhongxi Sunve (Shanghai, China). Cell Cycle Detection Kit was obtained from Keygene Biotech (Nanjing, China). L-Glutathione (GSH) and Nile red (NR) were purchased from Sigma-Aldrich (Diegem, Belgium). Fluorescein isothiocyanate (FITC), Rhodamine B Isothiocyanate (RITC), and buthionine sulfoximine (BSO) were purchased from Sigma (St. Louis, MO, USA). Cy5 labeled GAPDH molecular beacon (Cy5-GAPDH-MB, 5'-Cy5-CGACGGAGTCCTTCCACGATACCACGTCGDabcyl-3') was purchased from Sangon Biotech (Shanghai, China). Lipofectamine ${ }^{\mathrm{TM}} 2000$ and LysoTracker®Blue DND.22 was purchased from Invitrogen (Carlsbad, US). All of the other chemicals were of analytical or chromatographic grade.

\section{Cell culture}

MCF-7 (human breast carcinoma cell line) and MCF-7/Adr (multi-drug resistant variant) were provided by the Second Affiliated Hospital of Zhejiang University School of Medicine (Hangzhou, China). Cells were cultured in RMPI 1640 supplemented with 10\% FBS and $100 \mathrm{U} / \mathrm{ml}$ streptomycin-penicillin. For culture of MCF-7/ ADR cells, the medium was supplemented with $1.0 \mu \mathrm{g} /$ $\mathrm{ml}$ ADR.

\section{Synthesis of CS-ss-SA}

The CS-ss-SA was synthesized according to a literature method [16]. SA was conjugated with 3, 3' -dithiodipropionic acid mediated by dicyclohexylcarbodiimide (DCC) / dimethylaminopyridine (DMAP) in a molar ratio of $1: 1$. The reaction was performed at $60{ }^{\circ} \mathrm{C}$ for $24 \mathrm{~h}$ under an atmosphere of $\mathrm{N}_{2}$ and filtered to remove the byproducts. The intermediate product was activated by 1-ethyl-3-(3dimethylaminopropyl) carbodiimide (EDC) and added to the chitosan aqueous solution. Anhydrous DMSO was added (DMSO: $\mathrm{H}_{2} \mathrm{O}=8: 5$, molar ratio) to avoid precipitation. The mixture was stirred at $60{ }^{\circ} \mathrm{C}$ for the next $8 \mathrm{~h}$ and dialyzed against DI water for $48 \mathrm{~h}$ and centrifuged. The supernatant was lyophilized and rinsed using hot ethanol to remove the unreacted reagent. The product (CSss-SA) was re-dispersed in DI water and lyophilized. 


\section{Loading of PTX inside the cores of CS-ss-SA}

CS-ss-SA was dissolved in DI water and PTX/ ethanol solution was added $(20 \%, w / w)$ dropwise with constant stirring for $20 \mathrm{~min}$. The mixed solution was dialyzed against DI water overnight $(\mathrm{MWCO}=7000 \mathrm{Da})$ and the suspension was centrifuged at $4000 \mathrm{rpm}$ for $10 \mathrm{~min}$ to remove the unloaded PTX. The resulting supernatant was lyophilized and PTX loaded CS-ss-SA was obtained. The amount of PTX inside the cores was determined by a HPLC assay [17].

\section{Preparation of co-delivery complexes}

The CS-ss-SA/PTX/siBcl-2 co-delivery complexes were prepared by mixing the components at certain N/P ratios in DEPC-treated water. The particle size and zeta potential of the complexes were determined by DLS Spectrometer (Malvern Zetasizer).

\section{Gel retardation studies}

For gel retardation studies, CS-ss-SA/PTX/ siRNA co-delivery complexes with various $\mathrm{N} / \mathrm{P}$ ratios were subjected to electrophoresis on a $1.0 \%$ agarose gel containing ethidium bromide, using E-Gel electrophoresis system (Invitrogen) and visualized under UV light.

\section{RNase protection assay}

CS-ss-SA/siRNA complexes (containing $1 \mu \mathrm{g}$ siRNA) were incubated for $30 \mathrm{~min}$ at room temperature, followed by the addition of RNase A (10 U). As a control, free siRNA $(1 \mu \mathrm{g})$ was also treated with RNase I under the same condition. After 30 min incubation, the samples were further treated with RNase inhibitor (RI, $1 \mu \mathrm{L}=40$ $\mathrm{U})$ and incubation for $30 \mathrm{~min}$ at $37^{\circ} \mathrm{C}$ to terminate the activation of RNase $\mathrm{A}$, followed the addition of heparin $(0.05 \%)$ incubated for $30 \mathrm{~min}$. The integrity of siRNA was determined by gel electrophoresis at the same conditions as described before.

\section{Cell uptake and endo-lysosome escape studies}

The in vitro cell uptake and endo-lysosome escape of CS-ss-SA/siRNA complexes was determined by confocal studies. Cells were seeded $24 \mathrm{~h}$ prior to uptake in 12 -well plates containing cover slips at a density of $5 \times 10^{4}$ cells per well. CS-ss-SA/siRNA complexes were added to yield a final siRNA concentration of $100 \mathrm{nM}$. FAM-labeled siRNA (FAM-siRNA) was used as the model drug. After 1,4 , and $12 \mathrm{~h}$ of incubation, the media were replaced and stained with Lyso-tracker. Cells were subsequently rinsed with fresh PBS and fixed with $4 \%$ paraformaldehyde (PFA). The green fluorescence of complexes and blue fluorescence of endo-lysosome were visualized with the confocal microscope (Olympus IX81-FV1000).

\section{Intracellular triggered siRNA release}

A förster resonance energy transfer (FRET) assay was used to monitor the assembled structure and release dynamics of the complexes [18]. RITC-labeled CS-ss-SA solution was typically added to the FAM-siRNA solution at the desired N/P ratios to form FRET complexes. MCF7 cells were seeded into 12 -well plates containing cover slips at a density of $5 \times 10^{4}$ cells per well. After $24 \mathrm{~h}$ of plating, FRET complexes $(100 \mu \mathrm{L})$ were added. After incubation for $1,2,4$, and $12 \mathrm{~h}$, cells were rinsed with fresh PBS and fixed with 4\% PFA. FRET images of complexes were visualized by confocal microscopy (Nikon A1). An acceptor bleaching (AB) method was used to measure FRET efficiency by bleaching the acceptor and calculate the enhancement of the donor fluorescence [19]. The energy transfer efficiency ( FRET $_{\text {eff }}$ ) was quantified as:

$$
\mathrm{FRET}_{\text {eff }}=\left(\mathrm{D}_{\text {post }}-\mathrm{D}_{\text {pre }}\right) / \mathrm{D}_{\text {post }} \times 100 \%
$$

Consider that molecular beacon (MB) has a unique stem-loop configuration combined with fluorophore and quencher [20], we applied a Cy5-labeled GAPDHMB (Cy5-GAPDH-MB) as the oligonucleotide release indicator. CS-ss-SA/Cy5-GAPDH-MB complex was prepared by a similar method to the CS-ss-SA/siRNA complex. MCF-7 cells were seeded into 6-well plates at a density of $2 \times 10^{5}$ cells per well. After $24 \mathrm{~h}$ of plating, CS-ss-SA/Cy5-GAPDH-MB complex were added. After incubation for 1, 2, 4, and $12 \mathrm{~h}$, cells were rinsed with fresh PBS, collected and fixed with 4\% PFA. After centrifugation at $1200 \mathrm{rpm}$ for $5 \mathrm{~min}$ at $4{ }^{\circ} \mathrm{C}$ and wash with PBS twice, the cells were re-suspended in $0.5 \mathrm{~mL}$ of buffer. Flow cytometry (FC500MCL, Beckman Coulter) was used to measure the fluorescence intensity.

\section{Intracellular sequential drug delivery trafficking}

The fluorescence probe Nile red (NR) was utilized as the hydrophobic model drug and encapsulated in the CS-ss-SA/FAM-siNC complexes in accordance with the protocol. MCF-7 cells were seeded into 6-well plates containing cover slips at a density of $3 \times 10^{4}$ cells per well. After $24 \mathrm{~h}$ of plating, sequence-release complexes (CSss-SA/FAM-siRNA/NR) were added. After incubation for $4 \mathrm{~h}$, the medium was withdrawn and replaced with fresh medium. After 1, 4, 8, and $12 \mathrm{~h}$, cells were rinsed with fresh PBS and fixed with 4\% PFA. Fluorescence images of complexes were visualized by structured illumination microscopy (SIM).

\section{Bcl-2 silencing}

For gene silencing, MCF-7 cells were seeded into 6 -well plates at a density of $3 \times 10^{5}$ cells per well. After 24 $\mathrm{h}$ of plating, cells were incubated with CS-ss-SA/siBcl-2 
complexes with different siBcl-2 concentrations (50, 100, $200 \mathrm{nM})$. Lipofectamine $2000 / \mathrm{siBcl}-2$ (100nM) was used for control. RNA was extracted with TRIzol according to the standard protocol and total mRNA concentrations were detected by the nanodrop spectrophotometer. Reverse transcription was conducted with PrimeScript ${ }^{\mathrm{TM}}$ RT Reagent Kit (TaKaRa) in a $20 \mu \mathrm{L}$ SYBR ${ }^{\circledR}$ Green assay. Real-time PCR (StepOne, Applied Biosystems) was used to perform the amplification reaction. Bcl-2 primer sequence was 5'-GGATTGTGGCCTTCTTTGAG-3' and the reverse was 5'-TACCCAGCCTCCGTTATCCT-3'. The protocol was carried out for 40 cycles, comprising $95^{\circ} \mathrm{C}$ for $5 \mathrm{~s}$ and $60^{\circ} \mathrm{C}$ for $34 \mathrm{~s}$. GAPDH was also amplified as an internal control. A $\Delta \Delta \mathrm{CT}$ method was used for relative quantification of the expression levels. Each sample was performed in triplicate.

\section{Cell cycle analysis}

Co-delivery complexes (containing $100 \mathrm{nM}$ of siBcl-2 and $100 \mathrm{ng}$ of PTX) treated MCF-7 and MCF$7 /$ ADR cells $\left(2 \times 10^{6}\right.$ cells/well for 6 -well plate $)$ in $10 \%$ serum-containing culture medium were collected $48 \mathrm{~h}$ post-incubation and washed with ice cold PBS. The cells were fixed by ice cold $70 \%$ ethanol over night. After centrifugation at $1200 \mathrm{rpm}$ for $5 \mathrm{~min}$ at $4^{\circ} \mathrm{C}$ and wash with PBS twice, the cells were re-suspended in $0.5 \mathrm{~mL}$ of buffer containing RNase A and $50 \mathrm{mg} / \mathrm{mL}$ PI. All samples were measured by Beckman Coulter Cytomics FC-500 and cell cycle distributions were analyzed.

\section{Cytotoxicity assay}

In vitro cytotoxicity of the co-delivery complexes was evaluated by a cell viability assay. The MCF-7 and MCF-7/Adr cells were seeded into 96-well plates at a density of $5 \times 10^{4}$ cells per well. After $24 \mathrm{~h}$ of plating, cells were incubated with CS-ss-SA/siBcl-2/PTX complexes. After $48 \mathrm{~h}$ of incubation, MTT solution $(5.0 \mathrm{mg} / \mathrm{mL})$ was added and incubated for $4 \mathrm{~h}$. The culture medium was changed to DMSO to dissolve the purple formazan crystals. The absorbance at $570 \mathrm{~nm}$ was measured using a micro plate reader. The results indicated the cytotoxicity of co-delivery complexes was calculated.

\section{Statistical analysis}

All of the data represent the mean values \pm standard deviation of the independent measurements. Statistically significant differences between pairs of mean values were determined with ANOVA followed by Tukey-Kramer tests. Average deviation with p-values < 0.05 were considered statistically significant. Differences between groups were analyzed by Student's t-test, and mean differences with $\mathrm{p}$-values $<0.05$ were considered statistically significant.

\section{ACKNOWLEDGMENTS}

We appreciate the financial support from the National Natural Science Foundation of China (NSFC No. 81273442).

\section{CONFLICTS OF INTEREST}

The authors declare no competing financial interest.

\section{REFERENCES}

1. Eldar-Boock A, Polyak D, Scomparin A, Satchi-Fainaro R. Nano-sized polymers and liposomes designed to deliver combination therapy for cancer. Current opinion in biotechnology. 2013; 24:682-9. doi: 10.1016/j. copbio.2013.04.014.

2. Kemp JA, Shim MS, Heo CY, Kwon YJ. "Combo" nanomedicine: Co-delivery of multi-modal therapeutics for efficient, targeted, and safe cancer therapy. Advanced drug delivery reviews. doi: 2015.10.1016/j.addr.2015.10.019.

3. Qian X, Ren Y, Shi Z, Long L, Pu P, Sheng J, Yuan X, Kang C. Sequence-dependent synergistic inhibition of human glioma cell lines by combined temozolomide and miR-21 inhibitor gene therapy. Molecular pharmaceutics. 2012; 9:2636-45. doi: 10.1021/mp3002039.

4. Lee MJ, Ye AS, Gardino AK, Heijink AM, Sorger PK, MacBeath G, Yaffe MB. Sequential application of anticancer drugs enhances cell death by rewiring apoptotic signaling networks. Cell. 2012; 149:780-94. doi: 10.1016/j. cell.2012.03.031.

5. Hu CM, Zhang L. Nanoparticle-based combination therapy toward overcoming drug resistance in cancer. Biochemical pharmacology. 2012; 83:1104-11. doi: 10.1016/j. bcp.2012.01.008.

6. Wang Y, Gao S, Ye WH, Yoon HS, Yang YY. Co-delivery of drugs and DNA from cationic core-shell nanoparticles self-assembled from a biodegradable copolymer. Nature materials. 2006; 5:791-6. doi: 10.1038/nmat1737.

7. Zhao X, Li F, Li Y, Wang H, Ren H, Chen J, Nie G, Hao J. Co-delivery of HIF1alpha siRNA and gemcitabine via biocompatible lipid-polymer hybrid nanoparticles for effective treatment of pancreatic cancer. Biomaterials. 2015; 46:13-25. doi: 10.1016/j.biomaterials.2014.12.028.

8. Creixell M, Peppas NA. Co-delivery of siRNA and therapeutic agents using nanocarriers to overcome cancer resistance. Nano today. 2012; 7:367-79. doi: 10.1016/j. nantod.2012.06.013.

9. Taratula O, Garbuzenko OB, Chen AM, Minko T. Innovative strategy for treatment of lung cancer: targeted nanotechnology-based inhalation co-delivery of anticancer drugs and siRNA. Journal of drug targeting. 2011; 19:90014. doi: 10.3109/1061186X.2011.622404. 
10. He C, Lu K, Liu D, Lin W. Nanoscale metal-organic frameworks for the co-delivery of cisplatin and pooled siRNAs to enhance therapeutic efficacy in drug-resistant ovarian cancer cells. Journal of the American Chemical Society. 2014; 136:5181-4. doi: 10.1021/ja4098862.

11. Tsouris V, Joo MK, Kim SH, Kwon IC, Won YY. Nano carriers that enable co-delivery of chemotherapy and RNAi agents for treatment of drug-resistant cancers. Biotechnology advances. 2014; 32:1037-50. doi: 10.1016/j. biotechadv.2014.05.006.

12. Bartlett DW, Davis ME. Insights into the kinetics of siRNAmediated gene silencing from live-cell and live-animal bioluminescent imaging. Nucleic acids research. 2006; 34:322-33. doi: 10.1093/nar/gkj439.

13. Mao S, Sun W, Kissel T. Chitosan-based formulations for delivery of DNA and siRNA. Advanced drug delivery reviews. 2010; 62:12-27. doi: 10.1016/j.addr.2009.08.004.

14. Zhao MD, Hu FQ, Du YZ, Yuan H, Chen FY, Lou YM, Yu HY. Coadministration of glycolipid-like micelles loading cytotoxic drug with different action site for efficient cancer chemotherapy. Nanotechnology. 2009; 20:055102. doi: 10.1088/0957-4484/20/5/055102.

15. Zhao MD, Sun YM, Fu GF, Du YZ, Chen FY, Yuan $\mathrm{H}$, Zheng $\mathrm{CH}$, Zhang XM, Hu FQ. Gene therapy of endometriosis introduced by polymeric micelles with glycolipid-like structure. Biomaterials. 2012; 33:634-43. doi: 10.1016/j.biomaterials.2011.09.077.

16. Hu YW, Du YZ, Liu N, Liu X, Meng TT, Cheng BL, He JB, You J, Yuan H, Hu FQ. Selective redox-responsive drug release in tumor cells mediated by chitosan based glycolipid-like nanocarrier. Journal of controlled release. 2015; 206:91-100. doi: 10.1016/j.jconrel.2015.03.018.

17. You J, Li X, de Cui F, Du YZ, Yuan H, Hu FQ. Folateconjugated polymer micelles for active targeting to cancer cells: preparation, in vitro evaluation of targeting ability and cytotoxicity. Nanotechnology. 2008; 19:045102. doi: 10.1088/0957-4484/19/04/045102.

18. Lai J, Shah BP, Garfunkel E, Lee KB. Versatile fluorescence resonance energy transfer-based mesoporous silica nanoparticles for real-time monitoring of drug release. ACS nano. 2013; 7:2741-50. doi: 10.1021/nn400199t.

19. Qian J, Yao B, Wu C. Fluorescence resonance energy transfer detection methods: Sensitized emission and acceptor bleaching. Experimental and therapeutic medicine. 2014; 8:1375-80. doi: 10.3892/etm.2014.1928.

20. Zheng J, Yang R, Shi M, Wu C, Fang X, Li Y, Li J, Tan W. Rationally designed molecular beacons for bioanalytical and biomedical applications. Chemical Society reviews. 2015; 44:3036-55. doi: 10.1039/c5cs00020c.

21. Mansouri S, Cuie Y, Winnik F, Shi Q, Lavigne P, Benderdour M, Beaumont E, Fernandes JC. Characterization of folatechitosan-DNA nanoparticles for gene therapy. Biomaterials. 2006; 27:2060-5. doi: 10.1016/j.biomaterials.2005.09.020.
22. Liebmann JE, Hahn SM, Cook JA, Lipschultz C, Mitchell JB, Kaufman DC. Glutathione depletion by L-buthionine sulfoximine antagonizes taxol cytotoxicity. Cancer Res. 1993; 53:2066-2070.

23. Peng XH, Cao ZH, Xia JT, Carlson GW, Lewis MM, Wood WC, Yang L. Real-time detection of gene expression in cancer cells using molecular beacon imaging: new strategies for cancer research. Cancer Res. 2005; 65:1909-17. doi: 10.1158/0008-5472.CAN-04-3196.

24. Tsourkas A, Behlke MA, Xu Y, Bao G. Spectroscopic Features of Dual Fluorescence/Luminescence Resonance Energy-Transfer Molecular Beacons. Analytical Chemistry. 2003; 75:3697-703. doi: 10.1021/ac0342951.

25. Kelly PN, Strasser A. The role of Bcl-2 and its pro-survival relatives in tumourigenesis and cancer therapy. Cell death and differentiation. 2011; 18:1414-1424. doi: 10.1038/ cdd.2011.17.

26. Akar U, Chaves-Reyez A, Barria M, Tari A, Sanguino A, Kondo Y, Kondo S, Arun B, Lopez-Berestein G, Ozpolat B. Silencing of Bcl-2 expression by small interfering RNA induces autophagic cell death in MCF-7 breast cancer cells. Autophagy. 2014; 4:669-679. doi: 10.4161/auto.6083.

27. Karnak D, Xu L. Chemosensitization of prostate cancer by modulating Bcl-2 family proteins. Current drug targets. 2010; 11:699-707. doi: 10.2174/138945010791170888.

28. Patel MP, Masood A, Patel PS, Chanan-Khan AA. Targeting the Bcl-2. Curr Opin Oncol. 2009; 21:516-523. doi: 10.1097/CCO.0b013e328331a7a4.

29. Sternberg CN, Dumez H, Van Poppel H, Skoneczna I, Sella A, Daugaard G, Gil T, Graham J, Carpentier P, Calabro F, Collette L, Lacombe D, Grp EGTC. Docetaxel plus oblimersen sodium (Bcl-2 antisense oligonucleotide): an EORTC multicenter, randomized phase II study in patients with castration-resistant prostate cancer. Ann Oncol. 2009; 20:1264-1269. doi: 10.1093/annonc/mdn784.

30. Jang B, Kwon H, Katila P, Lee SJ, Lee H. Dual delivery of biological therapeutics for multimodal and synergistic cancer therapies. Advanced drug delivery reviews. doi: 2015.10.1016/j.addr.2015.10.023.

31. Zhang Z, Mei L, Feng SS. Paclitaxel drug delivery systems. Expert opinion on drug delivery. 2013; 10:325-40. doi: 10.1517/17425247.2013.752354.

32. Chou TC. Drug combination studies and their synergy quantification using the Chou-Talalay method. Cancer Res. 2010; 70:440-6. doi: 10.1158/0008-5472.CAN-09-1947.

33. Sun TM, Du JZ, Yao YD, Mao CQ, Dou S, Huang SY, Zhang PZ, Leong KW, Song EW, Wang J. Simultaneous delivery of siRNA and paclitaxel via a "two-in-one" micelleplex promotes synergistic tumor suppression. ACS nano. 2011; 5:1483-94. doi: 10.1021/nn103349h.

34. Khan M, Ong ZY, Wiradharma N, Attia AB, Yang YY. Advanced materials for co-delivery of drugs and genes in cancer therapy. Advanced healthcare materials. 2012; 1:373-92. doi: 10.1002/adhm.201200109. 
35. Biswas S, Deshpande PP, Navarro G, Dodwadkar NS, Torchilin VP. Lipid modified triblock PAMAMbased nanocarriers for siRNA drug co-delivery. Biomaterials. 2013; 34:1289-1301. doi: 10.1016/j. biomaterials.2012.10.024.
36. Greco F, Vicent MJ. Combination therapy: opportunities and challenges for polymer-drug conjugates as anticancer nanomedicines. Advanced drug delivery reviews. 2009; 61:1203-13. doi: 10.1016/j.addr.2009.05.006. 